certain to arouse controversy. $\mathrm{He}$ takes the extreme view that our present-day migration patterns are directly determined by natural selection. $\mathrm{He}$ also argues that natural selection is still acting in a similar fashion to that experienced by our hunter-gatherer ancestors. The argument is roughly as follows. In ancestral hunter-gatherers, newly formed pairs migrated away from their natal groups to other parts of the home range explored during adolescence. Pairs tended to settle in areas where their expected reproductive success (limited mainly by food supply) was highest. Males tended to explore larger ranges than females, partly because they could increase their reproductive success by incidental copulations on the way. Baker goes on to apply these speculative conclusions to industrial man: "I feel some justification in claiming that the migration pattern shown by modern industrial humans is that evolved during the 10-20 million years of hunting-gathering. All that remains is to consider whether the

\section{Inherited behaviour and reactions to drugs}

Drugs and the Inheritance of Behavior: A Survey of Comparative Psychopharmacogenetics. By P. L. Broadhurst. Pp. 206. (Plenum: New York and London, 1978.) £12.28.

P. L. BROADHURST has written a short but surprisingly complete book in the area of the effects of inherited behaviour on reactions to drugs. The title that he has taken was not his first choice and it is somewhat misleading. As everyone familiar with the field will know, this is a relatively small and new discipline which studies the effect of genotype on an organism's reaction to drugs. Almost all of the work discussed comes from rat and mouse experiments. The title implies that the drugs are altering the inheritance of behaviour, but of course the opposite is the case; it is the influence of inheritance on the response to drugs that is being studied. Dr Broadhurst would have preferred the title Psychopharmacogenetics but that title was pre-empted by B. E. Eleftheriou with an edited book (Plenum: New York, 1975). Others, such as McClearn, favour the name "Behavioural Pharmacogenetics" for the area.

Whatever the name, it is a field that has been entered on the one hand by behavioural geneticists often with insufficient knowledge of pharmacology, on the other by pharmacologists with insufficient knowledge of genetics or by psychologists with insufficient knowl- selection that is acting at the present time on modern industrial humans is tending to perpetuate or change the pattern." His highly controversial conclusion is that our present-day migration patterns are genetically controlled, hormonally mediated ("part of the physiological syndrome that transforms an individual to physical maturity is a decreasing migration threshold") behaviours. There is likely to be much argument against this conclusion. Even if one accepts in principle that genetic selection can still influence migration behaviour in man (a view for which no evidence is given), it is not at all clear to what extent factors such as extramarital copulation and protein intake alter inclusive fitness.

Although one may not agree with all of Baker's views, the book is thought provoking, and it will be a pity if it is not widely read because of its size and price.

John R. Krebs is Lecturer in Zoology at the Edward Grey Institute of Field Ornithology, Oxford, UK.

edge of both of the other two disciplines. Dr Broadhurst's book is replete with examples of the resultant chaos. He is properly critical of the investigators who have perpetrated some of the scientific monstrosities which often seem to have been undertaken with bad design, poor or nonexistent controls, poorly analysed and then published repeatedly.

There are, however, numerous examples of well designed and well executed studies. Students and those

\section{Aflatoxins}

Aflatoxins: Chemical and Biological Aspects. By J. G. Heathcote and J. R. Hibbert. Pp. 212. (Elsevier: Amsterdam and New York, 1978.) Dfl. 120; \$53.50.

MANY processed food components, and the foods themselves, are potential substrates for moulding by mycotoxinproducing fungi and so it is no small wonder that aflatoxin $\mathrm{B}$, being the most potent hepatocarcinogen in the rat, and related aflatoxins should be a subject of much biological and chemical research.

After opening chapters on the discovery of aflatoxins and their production under natural conditions and in the laboratory the chemistry of these substances is reviewed and their assay by physicochemical techniques and bioassay are described.

Thereafter, sufficient pathology is presented for the non-medical reader to appreciate the general nature of the effects without being blinded by technical jargon. The distinction between acute toxicity and carcinogenicity is made clear. just entering the field would do well to read this book and take heed of the lessons that it contains. It gives examples of the use of the most popular and powerful genetic techniques including selective breeding, sex differences, strain differences, diallel crosses and recombinant inbreeding.

For the novice to the field the precautions and controls that are outlined seem excessive and unnecessary; however, the experiments usually require months if not years of study and are often exceedingly expensive. The techniques are extremely powerful but much time and money can be wasted unless careful planning is done before rather than during or after the experiment.

The book has the decided advantage of being written by one individual with a consistent style and approach. It also has the advantage over edited books of being less self-serving and providing a broad overview rather than a series of narrow reports. In his desire to be complete, Dr Broadhurst has done a remarkable job, but therein also lies one of the criticisms of the book. In his zeal he has often used information contained in abstracts. Generally this is done because the data has not appeared in an edited journal. Thus, some of the conclusions reached by $\mathrm{Dr}$ Broadhurst rest on some rather thin ice.

Richard A. Deitrich

Richard A. Deitrich is Professor and ViceChairman of Pharmacology at the University of Colorado School of Medicine, and Research Associate of the Institute for Behavioral Genetics, Denver, Colorado.

The chapters on biochemistry and metabolism perhaps constitute the most intellectually stimulating feature of this book and the authors, who have worked in the field, have not hesitated to make certain value judgements. They are cautious concerning the significance of the elusive derivative aflatoxin $\mathbf{B}_{1}-2-3$ epoxide in acute toxicity and offer hemiacetal derivatives of aflatoxin as candidates for the role of carcinogen.

The book concludes with a well illustrated review of the biosynthesis of the aflatoxins and their interrelationships, followed by a chapter on control of aflatoxin contamination and current attempts at detoxification of foods.

The style of the text is clear and readable and typographical errors are rare. Those concerned with food science should read this book. I also suspect that the general reader could catch from it the fascination of aflatoxins and their mode of action.

\section{P. G. Mantle}

P. G. Mantle is Senior Tutor in Biochemistry at Imperial College, University of London, UK. 